\title{
Alterações bioquímicas e morfológicas após anastomose porto-cava e hepatectomia parcial. Estudo experimental em cães ${ }^{1}$
}

\author{
Valdinaldo Aragão de Melo ${ }^{2}$ \\ Reginaldo Ceneviva ${ }^{3}$ \\ Álvaro Queiroz de Godoy ${ }^{4}$ \\ José Ernesto dos Santos ${ }^{5}$ \\ Gustavo Barreto de Melo
}

\begin{abstract}
Melo VA, Ceneviva R, Godoy AQ, Santos JE, Melo GB. Alterações bioquímicas e morfológicas após anastomose porto-cava e hepatectomia parcial: estudo experimental em cães. Acta Cir Bras [serial online] 2003 Maio-Jun;18(3). Disponível em URL: http://www.scielo.br/acb.

RESUMO - Objetivo: Estudar os efeitos da anastomose porto-cava sobre a morfologia e bioquímica do fígado de cães. Métodos: Vinte animais foram divididos em 2 grupos: o Grupo I foi submetido à hepatectomia parcial de $28,7 \%$ e o Grupo II, à hepatectomia parcial associada à derivação portocava. Os parâmetros analisados foram: peso corpóreo, colesterol e triglicérides plasmáticos, lipídios e glicogênio hepáticos e índice ponderal de regeneração hepática. Os dados foram analisados no pré-operatório e no $30^{\circ}$ dia do pós-operatório. Resultados: No Grupo I, apenas os lipídios hepáticos estavam significativamente elevados 30 dias após a cirurgia. No Grupo II, o colesterol plasmático e o glicogênio hepático apresentaram redução no pós-operatório, enquanto os lipídios hepáticos estavam significativamente elevados. Comparando-se os 2 grupos, houve diferença estatisticamente significativa nos valores do colesterol plasmático, do glicogênio e do índice de regeneração hepática.

Conclusão: A anastomose porto-cava causa prejuízos morfológicos e bioquímicos significativos ao fígado, além de redução significativa do peso corpóreo.
\end{abstract}

DESCRITORES - Anastomose porto-cava. Hepatectomia parcial. Morfologia. Bioquímica.

\section{Introdução}

A anastomose porto-cava (APC) foi muito indicada como terapêutica efetiva na descompressão do sistema porta, reduzindo a ascite e controlando a hemorragia digestiva em pacientes com cirrose hepática ${ }^{1,2}$. Sua indicação, no entanto, foi restringida em conseqüência de diversas complicações pós-operatórias e pelos bons resultados da terapêutica endoscópica das varizes esofágicas. As principais complicações da APC são: atrofia do parênquima hepático, transtornos no metabolismo de carboidratos, lipídios e proteínas, encefalopatia porto-sistêmica e diminuição da capacidade de regeneração hepática ${ }^{3-7}$.

O parênquima hepático pode sofrer atrofia por redução do aporte de sangue portal ${ }^{8}$ ou pela perda dos fatores hepatotróficos conseqüente ao desvio do fluxo

1. Trabalho realizado na Faculdade de Medicina de Ribeirão Preto da Universidade de São Paulo.

2. Professor Adjunto do Departamento de Medicina da Universidade Federal de Sergipe.

3. Professor Titular do Departamento de Cirurgia e Anatomia da Faculdade de Medicina de Ribeirão Preto da Universidade de São Paulo.

4. Cirurgião do Hospital Evangélico de Londrina - PR.

5. Professor Associado do Departamento de Clínica Médica da Faculdade de Medicina de Ribeirão Preto da Universidade de São Paulo.

6. Acadêmico de Medicina da Universidade Federal de Sergipe. 
portal $^{9}$. Entre outros fatores, os elevados níveis da amônia sangüínea, decorrentes da metabolização hepática reduzida, causam a encefalopatia hepática. Ela é tanto mais grave quanto mais intensa for a lesão prévia do fígado $2,5,10$.

A necessidade de se compreender melhor as alterações causadas pela anastomose porto-cava justifica novas investigações. O objetivo deste trabalho é estudar os efeitos da APC sobre a morfologia e bioquímica do fígado de cães submetidos a esse procedimento.

\section{Ética}

O presente trabalho foi realizado de acordo com as recomendações da Declaração de Helsinque, com as Normas Internacionais de Proteção aos Animais e com o Colégio Brasileiro de Experimentação Animal.

\section{Métodos}

Foram utilizados 20 cães adultos, mestiços, do sexo masculino, pesando entre 13 e $20 \mathrm{~kg}$, provenientes do Biotério Central da Faculdade de Medicina de Ribeirão Preto da Universidade de São Paulo.

Eles foram divididos em 2 grupos:

- Grupo I: constituído de 10 cães submetidos à hepatectomia parcial, em média de $28,7 \%$, mediante ressecção do lobo lateral esquerdo. Este grupo serviu como controle.

- Grupo II: constituído de 10 cães submetidos à hepatectomia parcial, em média de $28,7 \%$, mediante ressecção do lobo lateral esquerdo, e APC términolateral.

No dia da cirurgia, os animais foram mantidos com água ad libitum. Nembutal sódico (etil-1 metilbutil-barbiturato sódico) na dose de $30 \mathrm{mg} / \mathrm{kg}$ de peso corpóreo foi aplicado endovenosamente e complementado quando se percebia superficialização da anestesia. A respiração foi controlada com respirador automático e cânula traqueal.

Após tricotomia e antissepsia da região abdominal anterior, realizou-se laparotomia com incisão mediana longitudinal xifo-umbilical. Posteriormente, ressecouse, nos 2 grupos, o lobo lateral esquerdo depois de dissecção adequada dos ligamentos e do hilo hepático. No Grupo II, além do que foi descrito, foi feita APC término-lateral. Para tanto, a veia cava inferior foi dissecada desde a veia renal direita até o segmento retrohepático. Os pequenos vasos lombares foram ligados. A veia porta também foi dissecada desde a esplênica até sua bifurcação. Após pinçamento de ambas as veias, a veia porta foi seccionada, com ligadura do coto distal, junto ao fígado, e o proximal, anastomosado à cava inferior. Finalizado esse procedimento, a cavidade abdominal foi fechada mediante sutura da parede por planos.

No primeiro dia pós-operatório, os animais receberam dieta líquida e, a partir do segundo, alimentos sólidos foram liberados. Trinta dias depois da cirurgia, os cães foram submetidos à retirada de sangue venoso após 12 horas de jejum. Posteriormente, foram sacrificados com o uso de nembutal sódico até parada cárdio-respiratória.

Os parâmetros utilizados foram os seguintes:

1. Peso corpóreo $(\mathrm{kg})$ : os animais foram pesados antes e 30 dias depois da cirurgia.

2. Dosagem de colesterol plasmático ( $\mathrm{mg} / \mathrm{dl})$ : foi determinado no período pré-operatório e no $30^{\circ}$ dia do pós-operatório.

3. Dosagem dos triglicérides plasmáticos (mg/dl): foram analisados antes e 30 dias após a cirurgia.

4. Quantificação de lipídios no tecido hepático ( $\mathrm{g} \%$ ): o fígado retirado na hepatectomia e no sacrifício após 30 dias foi utilizado para essa dosagem.

5. Quantificação do glicogênio hepático ( $\mathrm{g} \%)$ : analisado com a amostra de fígado retirada na cirurgia e no sacrifício.

6. Índice ponderal de regeneração hepática (IR): a partir do lobo lateral esquerdo ressecado (LLE), equivalente a $28,7 \%$ do total, foi calculado o peso do fígado remanescente (FR). O fígado ressecado e pesado por ocasião do sacrifício correspondeu ao peso do fígado à necropsia $(\mathrm{FN})$. $\mathrm{O}$ índice de regeneração hepática foi calculado pela formula de $\mathrm{CHILD}^{11}$ : IR = (FN - FR)/LLE x 100. Quando a diferença entre o peso do fígado à necropsia e o peso do fígado remanescente calculado era igual ao removido durante a cirurgia, o índice de regeneração correspondia a $100 \%$.

Para a análise estatística dos resultados que envolveram até duas observações, como pré e pósoperatório, utilizou-se o teste de Wilcoxon e nos que envolveram mais de duas observações, o teste de Friedman. Para as comparações de dois grupos diferentes de procedimentos, foi utilizado o teste de Mann-Whitney. O nível de significância considerado foi de 0,05 .

\section{Resultados}

Os resultados pré e pós-operatórios do peso corpóreo, colesterol plasmático, triglicérides, lipídios e glicogênios hepáticos dos Grupos I e II estão expressos, respectivamente nas tabelas 1 e 2 . 
TABELA 1 - Resultados do Grupo I.

\begin{tabular}{l|c|c|c}
\hline & Pré-operatório & Pós-operatório (30 dias) & Estatística \\
\hline Peso corpóreo & $14,8 \pm 2,1$ & $5 \pm 2,6$ & \\
\hline Colesterol plasmático & $134,4 \pm 23,3$ & $124,9 \pm 26,1$ & \\
\hline Triglicérides & $73 \pm 10,2$ & $67,9 \pm 11,2$ & \\
\hline Lipídios hepáticos & $2,7 \pm 0,8$ & $3,3 \pm 0,6$ & $\mathrm{p}<0,05$ \\
\hline Glicogênio hepático & $3,3 \pm 1,4$ & $3,1 \pm 2,3$ & \\
\hline
\end{tabular}

TABELA 2 - Resultados do Grupo II.

\begin{tabular}{l|c|c|c}
\hline & Pré-operatório & Pós-operatório (30 dias) & Estatística \\
\hline Peso corpóreo & $18 \pm 1,6$ & $15,1 \pm 2,5$ & $\mathrm{p}<0,01$ \\
\hline Colesterol plasmático & $111,1 \pm 23,4$ & $79 \pm 16$ & $\mathrm{p}<0,05$ \\
\hline Triglicérides & $68,7 \pm 7,7$ & $56 \pm 13,8$ & \\
\hline Lipídios hepáticos & $2,9 \pm 0,6$ & $4,3 \pm 1,5$ & $\mathrm{p}<0,05$ \\
\hline Glicogênio hepático & $2,8 \pm 2,2$ & $0,27 \pm 0,29$ & $\mathrm{p}<0,05$ \\
\hline
\end{tabular}

Não houve diferença do peso corpóreo dos animais do Grupo I antes e 30 dias após o procedimento cirúrgico. Nos animais do Grupo II, houve perda de peso no pós-operatório estatisticamente significativa.

Entre os cães do grupo controle, o colesterol plasmático não estava alterado 30 dias após a cirurgia. Nos animais do grupo submetido à anastomose portocava, houve redução significativa no mesmo período. Diferença estatística também foi encontrada quando se compararam os dois grupos no período pósoperatório $(\mathrm{p}<0,01)$.

Em relação aos valores de triglicérides plasmáticos, não houve diferença estatisticamente significativa entre os períodos pós e pré-operatórios nos animais em cada grupo e entre os dois grupos.

$\mathrm{Na}$ quantificação dos lipídios, houve diferença estatística entre os períodos analisados em ambos os grupos de animais. Quanto ao glicogênio, foi constatada diferença entre o grupo controle e o com APC 30 dias após a cirurgia $(\mathrm{p}<0,001)$.

O índice ponderal de regeneração hepática apresentou diferença estatisticamente significativa entre os dois grupos de cães, com um valor de 112,3 $\pm 54,7 \%$ para o Grupo I e de $-21,2 \pm 28,4 \%$ para o Grupo II.

\section{Discussão}

A derivação do sangue portal para a veia cava inferior foi idealizada no final do século XIX como opção terapêutica para descompressão do sistema porta em presença de hipertensão portal ${ }^{12}$. Entretanto, após a descoberta de várias complicações pós-operatórias, esse procedimento passou a ser substituído pelas derivações seletivas e cirurgias não-derivativas ${ }^{10,13}$.

Entre as complicações do desvio do sangue portal, cita-se a alteração do estado nutricional, avaliado na maioria dos casos pela modificação do peso corpóreo e taxas de proteínas plasmáticas ${ }^{14,15}$.

Em alguns trabalhos, foi relatado redução de $25 \%$ de peso corpóreo após um mês de observação e 28,3\% depois de cinco meses ${ }^{14,15}$. Os resultados obtidos neste trabalho foram concordantes com os descritos na literatura. Observou-se redução de $16,1 \%$ no peso corpóreo dos animais submetidos à derivação portocava, enquanto os submetidos apenas à hepatectomia parcial não apresentaram modificação.

A explicação mais adequada para essa alteração é que a exclusão do fígado do sangue portal altera significativamente o metabolismo protéico, conduzindo o animal a um estado de desnutrição protéica progressiva $^{14}$.

Foi observada redução significativa do colesterol plasmático nos animais com APC, enquanto os triglicérides não apresentaram alterações significativas nos dois grupos.

Observando dados encontrados na literatura, podese notar que a APC diminui a massa hepática e síntese de colesterol ${ }^{16,17}$. Esse tipo de derivação portal já foi utilizado para o tratamento de hipercolesterolemia familial no homem, promovendo redução do colesterol 
plasmático de 30 a 40\%. Atualmente, no entanto, o transplante hepático é considerado a opção terapêutica mais adequada ${ }^{17}$.

A avaliação bioquímica do tecido hepático incluiu a dosagem dos lipídios totais e do glicogênio. Os primeiros aumentaram significativamente após hepatectomia parcial isolada ou com anastomose portocava, tendo nesta o efeito mais acentuado. Uma explicação para esse acúmulo de gordura seria a redução de lipoproteínas pelo fígado ou a diminuição de sua capacidade de oxidação dos ácidos graxos ${ }^{18}$.

As dosagens de glicogênio hepático foram feitas após 12 horas de jejum e houve uma redução para um décimo do valor inicial nos animais submetidos à derivação do sangue portal. $\mathrm{O}$ mecanismo pelo qual isso ocorre é desconhecido.

Bismuth e col. ${ }^{19}$ observaram o aumento da concentração periférica da insulina após a anastomose porto-cava, promovendo maior utilização periférica de glicose. Esse aumento parece ser o responsável pela correção da glicemia e tolerância ao jejum no paciente com glicogenose após APC. A glicose não seria captada e estocada no fígado sob a forma de glicogênio não utilizável.

O índice de regeneração hepática demonstrou uma recuperação ponderal total da massa hepática após a hepatectomia parcial em observação de um mês. O sangue portal tem grande importância na regeneração hepática, por carrear fatores hepatotróficos importantes para a adequada regeneração ${ }^{8,9}$.

A regeneração hepática, tendo como parâmetro a massa hepática, estava reduzida nos animais com derivação porto-cava após um mês de observação.

Sabe-se que o desvio do sangue portal diminui o débito sangüíneo hepático total, privando-o do oxigênio e fatores hepatotróficos provenientes da veia porta e necessários aos processos metabólicos do fígado ${ }^{20}$. A restauração desse fluxo previne a atrofia hepática ${ }^{21}$.

\section{Conclusão}

A anastomose porto-cava causa prejuízos morfológicos e bioquímicos significativos ao fígado, além de redução significativa do peso corpóreo.

\section{Referências}

1. Child III CG. Eck's fistula. Surg Gynecol Obstet 1953;96:375.

2. Franco D, Castaing D, Bismuth H. Conséquences métaboliques de dérivations portales expérimentales. Pathol Biol 1979;27(4):192-6.

3. Resnick RH, Iber FL, Ishirara AM, Chalmers TC, Zimmerman H. A controlled study of the therapeutic portocaval shunt. Gastroenterology 1974;67(5):843-57.
4. Pezzarossa A, Contini S, Bonora E, Marni A, Colotto G, Capocasale E, Butturine U. Glucose tolerance after portacaval shunt in liver cirrhosis. Diabete Metab 1986;12(4):197-202.

5. Meyer HP, Legemate DA, van den Brom W, Rothuizen J. Improvement of chronic hepatic encephalopathy in dogs by the benzodiazepine-receptor partial inverse agonist sarmazenil, but not by the antagonist flumazenil. Metab Brain Dis 1998;13(3):241-51.

6. Awad SS, Hemmila MR, Soldes OS, Sawada S, Rich PB, Mahler S, Gargulinski M, Hirschl RB, Bartlett RH. A novel reproducible model of hepatic failure in canines. J Surg Res 2000;94(2): 167-71.

7. Ennker IC, Mei M, Nagel E, Hauss J, Reiss G, Oellerich M, Pichlmayr R. Changes in liver function and morphology after various portocaval bypass operations. Langenbecks Arch Chir 1992;377(3):144-51.

8. Sato Y, Tsukada K, Tanaka K, Hatakeyama K. New aspects in the liver regeneration following partial hepatectomy. Nippon Geka Gakkai Zasshi 1997;98:706-12.

9. Richardson PD, Withrington PG. Physiological regulation of the hepatic circulation. Ann Rev Physiol 1982;44:57-69.

10. Antunes RA, Duval-Araujo I. Evolução das varizes esofagogástricas após anastomose esplenorrenal proximal versus esplenorrenal distal. Rev Col Bras Cir 2000;27(2): 102-8.

11. Child CG, Barr D, Holswade GR, Harrisons CS. Liver regeneration following portocaval transposition in dog. Ann Surg 1953;138:600.

12. Eck NV. Kvoprosu o perevyaskie vorotnois veni. Predvaritennoye soobshtshjenys (Ligature of the portal vein). Voen Med J 1877;130:1.

13. Zacks SL, Sandler RS, Biddle AK, Mauro MA, Brown RS Jr. Decision-analysis of transjugular intrahepatic portosystemic shunt versus distal splenorenal shunt for portal hypertension. Hepatology 1999;29:1399-1405.

14. Goffi FS. Alguns aspectos metabólicos experimentais das derivações porto-cavas simples ou associadas a derivação aortoportal [Tese - Livre Docência]. Universidade de São Paulo; 1955 .

15. Adamsons RJ, Arif A, Babibh A, Butt K, Lam A, Minkowitz S. Arterialization of the liver in combination with a portocaval shunt in the dog. Surg Gynecol Obstet 1975;140(4):594-600.

16. Starzl TE, Porter KA, Kashiwagi N. Portal hepatotrophic factors, diabetes mellitus and acute liver atrophy, hypertrophy and regeneration. Surg Gynecol Obstet 1975;141(6):843-58.

17. Lopez-Santamaria M, Migliazza L, Gamez M, Murcia J, DiazGonzalez M, Camarena C, Hierro L, De la Vega A, Frauca E, Diaz M, Jara P, Tovar J. Liver transplantation in patients with homozygotic familial hypercholesterolemia previously treated by end-to-side portocaval shunt and ileal bypass. J Pediatr Surg 2000;35(4):630-3.

18. Feigelson EB, Pfaff WW, Karmen A, Steinberg D. The role of plasma free fatty acids in development of fatty liver. J Clin Invest 1961;40:2171.

19. Bismuth H, Franco D, Grande D. Les dérivations portales dans les maladies métaboliques du foie. Gastroenterol Clin Biol 1977;1(11):903-16.

20. Fisher B, Fisher ER, Lee S. Experimental evaluation of liver atrophy and portocaval shunt. Surg Gynecol Obstet 1967;125(6): 1253-8.

21. Adamsons RJ, Butt K, Iyer S, De Rose J, Dennis CR, Kinkhabwala M, Gordon D, Martin E. Portacaval shunt with arterialization of the portal vein means of a low arterio-venous fistula. Surg Gynecol Obstet 1978;146(6):869-76. 
Melo VA, Ceneviva R, Godoy AQ, Santos JE, Melo GB. Biochemical and morphological alterations after portocaval shunt and partial hepatectomy: experimental study in dogs. Acta Cir Bras [serial online] 2003 May-Jun;18(3). Available from URL: http://www.scielo.br/acb.

ABSTRACT - Purpose: The aim of this study was to assess the effect of portocaval shunt on liver biochemical and morphological aspects in dogs. Methods: Twenty animals were divided into 2 groups: Group I was submitted to $28.7 \%$ partial hepatectomy and Group II underwent partial hepatectomy associated with portocaval shunt. The data analyzed were: body weight, serum cholesterol and triglyceride, hepatic lipids and glycogen and liver regeneration index. All these data were measured preoperatively and 30 days after surgery. Results: In Group I, only hepatic lipids levels were statistically increased post-operatively. In Group II, serum cholesterol and hepatic glycogen values were reduced while hepatic lipids levels were increased 30 days after surgery. When both groups were compared to each other, serum cholesterol, hepatic glycogen and liver regeneration index showed statistically significant difference. Conclusion: Portocaval shunt causes morphological and biochemical impairment of the liver as well as reduction of body weight.

KEY WORDS - Portocaval shunt. Partial hepatectomy. Biochemistry. Morphology.

Conflito de interesse: nenhum

Correspondência:

Fonte de financiamento: nenhuma

Valdinaldo Aragão de Melo

Rua Álvaro Brito, 14/30

49020-400 Aracaju - SE

Tel: (79)246-2217 - Fax: (79_246-2857

vamelo@infonet.com.br

Data do recebimento: $19 / 12 / 2002$

Data da revisão: 28/12/2002

Data da aprovação: 07/01/2003 